\title{
A Proposed Technique for Solving Quasi-Concave Quadratic Programming Problems with Bounded Variables by Objective Separable Method
}

\author{
M. Asadujjaman* and M. Babul Hasan \\ Department of Mathematics, Dhaka University, Dhaka-1000, Bangladesh
}

(Received: 8 July 2015; Accepted: 8 December 2015)

\begin{abstract}
In this paper, a new method namely, objective separable method based on Linear Programming with Bounded Variables Algorithm is proposed for finding an optimal solution to a Quasi-Concave Quadratic Programming Problems with Bounded Variables in which the objective function involves the product of two indefinite factorized linear functions and the constraint functions are in the form of linear inequalities. For developing this method, we use programming language MATHEMATICA. We also illustrate numerical examples to demonstrate our method.
\end{abstract}

Keywords: Quadratic Programming, Quasi-Concave Quadratic Programming, Bounded Variable, Lower \& Upper Bound, Objective separable.

\section{Introduction}

Non-linear programming (NLP) is the process of solving an optimization problem defined by a system of equalities and inequalities, collectively termed constraints, over a set of unknown real variables, along with an objective function to be maximized or minimized, where some of the constraints or the objective function is non-linear. It is the sub-field of mathematical optimization that deals with problems that are not linear. A quadratic programming (QP) is a special type of mathematical optimization problem. It is the problem of optimizing (minimizing or maximizing) a quadratic function of several variables subject to linear constraints on these variables. Because of its usefulness in Producing Planning, Financial and Corporate Planning, Health Care and Hospital Planning and Engineering, QP is viewed as a discipline in Operational Research and it has become a fertile area in the field of research in recent years. A large number of algorithms for solving QP problems have been developed. Some of them are extensions of the simplex method and others are based on different principles. In the conversance, a great number of methods (Wolf $^{1}$, Beale $^{2}$, Frank and Wolf ${ }^{3}$, Shetty ${ }^{4}$, Lemke $^{5}$, Best and Ritter $^{6}$, Theil and van de Panne ${ }^{7}$, Boot ${ }^{8}$, Fletcher ${ }^{9}$, Swarup $^{10}$, Gupta and Sharma ${ }^{11}$, Moraru ${ }^{12,13}$, Jensen and King ${ }^{14}$, Bazaraa, Sherali and Shetty ${ }^{15}$ ) are designed to solve QP problems in a finite number of steps. Among them, Wolf's method $^{1}$, Swarup's simplex method ${ }^{10}$ and Gupta and Sharma's method ${ }^{11}$ are more popular than the other methods. The above mentioned articles deal with variables of the type $\geq 0$ but no upper bound. But when considering real-world applications of QP, it may arrive that one or more unknown variables $x_{j}$ not only have a non-negativity restriction but also have upper and lower bounds on them. In this case, the above mentioned articles did not consider the upper bounds on the variables. Andrew Whinston ${ }^{16}$ developed a method for solving QP problems with bounded variables but not consider quasi-concave QP problems with bounded variables.

In this paper, we proposed a new method namely, objective separable method (OSM) based on linear programming with bounded variables (LPBV) algorithm for finding an optimal solution to a quasi-concave QP problems with bounded variables in which the objective function involving the product of two indefinite factorized linear functions and constraints functions are in the form of linear inequalities. In this proposed method, we construct two linear programming problems with bounded variables both of maximization type from the given quasi-concave QP problems with bounded variables. Then we obtain an optimal solution to the given quasi-concave QP problems with bounded variables from the solutions of the two constructed linear programming problems. The OSM based on LPBV algorithm which differs from the existing methods (Wolf's method ${ }^{1}$, Swarup's simplex method ${ }^{10}$ and Gupta and Sharma's $\operatorname{method}^{11}$ ). We use the concept of LPBV method to solve this problem. For developing this method, use programming language MATHEMATICA. We also illustrate numerical examples to demonstrate our method.

The rest of the paper is organized as follows. In Section II, we discuss on glossary background of LPBV, QP, quasi-concave QP, Gonzi property and some results. In Section III, we discuss quasi-concave QPBV problems and constructed two single objective linear programming problems from the problem (P). In Section IV, we discuss the existing method and existing algorithm for LPBV problems. In Section $\mathrm{V}$, we discuss our proposed algorithm for quasi-concave QPBV problems and illustrate the solution procedure with a number of numerical examples. In Section VI, we develop a computer technique for this method by using progra-mming language MATHEMATICA and solve the previous examples through the computer technique. Finally, we draw a conclusion in Section VII.

\section{Preliminaries}

In this section, we briefly discuss definitions of LPBV, $\mathrm{QP}$, quasi-concave QP, Gonzi property and some results.

\section{Gonzi Property}

Let $f_{1}(x)$ and $f_{2}(x)$ be two differentiable functions on $X \subset R^{n}$, an $n$-dimensional Euclidean space. The functions $f_{1}(x)$ and $f_{2}(x)$ are said to have the Gonzi property ${ }^{23}$ in $X \subset R^{n}$ if

$$
\left(f_{1}(x)-f_{1}(u)\right)\left(f_{2}(x)-f_{2}(u)\right) \leq 0, \forall x, u \in X
$$




\section{Theorem 2.1}

The product $f_{1}(x) f_{2}(x)$ of two linear functions $f_{1}(x)$ and $f_{2}(x)$ is concave iff the functions $f_{1}(x)$ and $f_{2}(x)$ have the Gonzi property.

\section{LPBV Problems}

In Linear Programming (LP) models ${ }^{17}$, variables may have explicit positive upper and lower bounds. For example, in production facilities, lower and upper bounds can represent the minimum and maximum demands for certain products. Bounded variables also arise prominently in the course of solving integer programming problems by the branch and bound algorithm.

Consider the following LP problems,

$$
\begin{array}{ll}
\text { Maximize, } & Z=C X \\
\text { Subject to, } & (A, I) X=b \\
& L \leq X \leq U
\end{array}
$$

where, $U=\left(\begin{array}{c}u_{1} \\ u_{2} \\ \vdots \\ u_{n+m}\end{array}\right) \& L=\left(\begin{array}{c}l_{1} \\ l_{2} \\ \vdots \\ l_{n+m}\end{array}\right), U \geq L \geq 0$.

The elements of $L$ and $U$ for an unbounded variable 0 and $\infty$.

\section{QP Problems}

The general QP problem can be written as

$$
\begin{array}{ll}
\text { Maximize, } & Z=c x+\frac{1}{2} x^{T} Q x \\
\text { Subject to, } & A x \leq b \text { and } x \geq 0
\end{array}
$$

Where $c$ is an $n$-dimensional row vector describing the coefficients of the linear terms in the objective function, and $Q$ is an $(n \times n)$ symmetric real matrix describing the coefficients of the quadratic terms. If a constant term exists it is dropped from the model. As in LP, the decision variables are denoted by the $n$-dimensional column vector $x$, and the constraints are defined by an $(m \times n) A$ matrix and an $m$-dimensional column vector $b$ of right-hand side coefficients. We assume that a feasible solution exists and that the constraints region is bounded. When the objective function $Z$ is strictly convex for all feasible points the problem has a unique local maximum which is also the global maximum. A sufficient condition to guarantee strictly convexity is for $Q$ to be positive definite.

\section{Quasi-Concave QP Problems}

In this paper, we consider the quasi-concave QPBV problems subject to linear constraints.

The quasi-concave QP problems ${ }^{18}$ can be written as

$$
\begin{aligned}
& \text { Maximize, } \quad Z=(c x+\alpha)(d x+\beta) \\
& \text { Subject to, } \quad A x \leq b \text { and } x \geq 0
\end{aligned}
$$

where, $A$ is an $(m \times n)$ matrix, $b \in \mathfrak{R}^{m}$, and $x, c, d \in \mathfrak{R}^{n}$ and $\alpha, \beta \in \Re$. Here we assume that (i) $(c x+\alpha)$ and $(d x+\beta)$ have Gonzi property in feasible set.

(ii) The constraints set $S=\{x: A x=b, x \geq 0\}$ is nonempty and bounded.

\section{Quasi-Concave QPBV Problems}

Let us consider the general quasi-concave QPBV problem

(P) $\operatorname{Max}, Z=z^{1}(x) \cdot z^{2}(x)$

$$
=\left(\sum_{j=1}^{n} c_{j} x_{j}+\alpha\right) \cdot\left(\sum_{j=1}^{n} d_{j} x_{j}+\beta\right)
$$

Subject to,

$$
\begin{gathered}
\sum_{j=1}^{n} a_{i j} x_{j}=b_{i}, \quad i=1,2, \cdots \cdots, m \\
l_{j} \leq x_{j} \leq u_{j}, \quad j=1,2, \cdots \cdots, n
\end{gathered}
$$

where, $l_{j} \leq u_{j}, j=1,2, \cdots \cdots \cdots, n$. Here $l_{j}$ and $u_{j}$ are usually called lower-bound and upper-bound of the constraints.

Let us assume that $z^{1}(x), z^{2}(x)$ have the Gonzi property in the feasible set for all

$x=\left(x_{1}, x_{2}, \cdots \cdots, x_{m}, x_{m+1}, \cdots \cdots, x_{n}\right)^{T} \in S, \quad$ where $S$ denotes a feasible set defined by the constraints (4) and (5). Also assume that $S$ is non-empty and bounded. Thus, by the Theorem 2.1 , it is concluded that the problem $(\mathrm{P})$ is a concave non-linear programming problem with bounded variables in which the objective function involving the product of two indefinite factorized linear functions and constraints functions are in the form of linear inequalities. This implies that the optimal solution of the problem (P) exists and it occurs at an extreme point of the feasible region.

Now, from the problem (P), two single objective linear programming problems are constructed as follows:

(P1) $\operatorname{Max}, z^{1}(x)$

$$
=\left(\sum_{j=1}^{n} c_{j} x_{j}+\alpha\right)
$$

Subject to,

$$
\begin{aligned}
& \sum_{j=1}^{n} a_{i j} x_{j}=b_{i}, \quad i=1,2, \cdots \cdots, m \\
& l_{j} \leq x_{j} \leq u_{j}, \quad j=1,2, \cdots \cdots, n
\end{aligned}
$$

where, $l_{j} \leq u_{j}, j=1,2, \cdots \cdots \cdots, n$. Here $l_{j}$ and $u_{j}$ are usually called lower-bound and upper-bound of the constraints.

(P2) $\operatorname{Max}, z^{2}(x)$

$$
=\left(\sum_{j=1}^{n} d_{j} x_{j}+\beta\right)
$$

Subject to,

$$
\begin{aligned}
& \sum_{j=1}^{n} a_{i j} x_{j}=b_{i}, \quad i=1,2, \cdots \cdots, m \\
& l_{j} \leq x_{j} \leq u_{j}, \quad j=1,2, \cdots \cdots, n
\end{aligned}
$$


where, $l_{j} \leq u_{j}, j=1,2, \cdots \cdots \cdots, n$. Here $l_{j}$ and $u_{j}$ are usually called lower-bound and upper-bound of the constraints.

\section{Remark 3.1}

From above, we can easily conclude that if both (P1) and $(\mathrm{P} 2)$ are solvable, then the problem $(\mathrm{P})$ is solvable.

Now, the following theorem connecting the optimal solutions of the problem $(\mathrm{P})$, the problem $(\mathrm{P} 1)$ and the problem $(\mathrm{P} 2)$ which is used in the proposed method.

\section{Theorem 3.1}

Let $X_{0}$ be an optimal solution to the problem (P1). If $\left\{X_{n}\right\}$ is a sequence of basic feasible solutions to the problem (P2) by linear programming with bounded variables algorithm considering the solution $X_{0}$ as an initial feasible solution such that $Z\left(X_{k}\right) \leq Z\left(X_{k+1}\right)$ for all $k=0,1,2, \cdots \cdots, n$ and either $X_{n+1}$ is an optimal solution to the problem (P2) or $Z\left(X_{n+1}\right) \geq Z\left(X_{n+2}\right)$, then $X_{n+1}$ is an optimal solution to the problem $(\mathrm{P})^{22}$.

\section{Existing Method for LPBV Problems}

In this section, we discuss the existing method ${ }^{17}$ and existing algorithm ${ }^{17,19}$ for LPBV problems.

One can solve LPBV problems by regular simplex method by considering the lower and upper bound constraints explicitly which is not computationally efficient as the number of constraints as well as the number of variables become large and studied LP problems with upper bounded variables, which uses smaller basis to solve LPBV problems. In which case, from (1) and (2), the constraints are put in the form,

$$
\begin{gathered}
(A, I) X=b \\
X+X^{\prime}=U \\
X-X^{\prime \prime}=L \\
X, X^{\prime}, X^{\prime \prime} \geq 0
\end{gathered}
$$

Where $X^{\prime}$ and $X^{\prime \prime}$ are slack and surplus variables. This problem includes $3(m+n)$ variables and $(3 m+2 n)$ constraints equations. However, the size can be reduced considerably through the use of special techniques that ultimately reduce the constraints to the set $(A, I) X=b$.

First, we consider the lower-bounds. Given $X \geq L$, we can use the substitution $X=L+X^{\prime \prime}, \quad X^{\prime \prime} \geq 0, \quad L \geq 0$. Throughout and solve the problem in terms of $X^{\prime \prime}$. The original $X$ is determined by back-substitution which is legitimate because it guarantees that $X=L+X^{\prime \prime}$ will remain non-negative for all $X^{\prime \prime} \geq 0$. Next, we consider the upper-bounding constraints, $X \leq U$. The idea of direct substitution (i.e. $X=U-X^{\prime}, X^{\prime} \geq 0$ ) is not correct because back substitution, $X=U-X^{\prime}$, does not ensure that $X$ will remain non-negative. This difficulty is over come by using a simplex method variation that accounts for the upper bounds implicitly. Define the upper bounded LP model as

$$
\text { Maximize, } Z=\{C X \mid(A, I) X=b, 0 \leq X \leq U\}
$$

The bounded primal simplex method uses only the constraints $(A, I) X=b, X \geq 0$, while accounting for $X \leq U$ implicitly by modifying the simplex feasibility condition. Let $X_{B}=B^{-1} b$ be a current basic feasible solution of $(A, I) X=b, X \geq 0$ and suppose that according to the regular optimality condition, $P_{j}$ is the entering vector. In developing the new feasibility condition, two main points must be considered. First one, the non-negativity and upper-bound constraints for the entering variable and secondly, for those basic variables that may be affected by introducing the entering variables.

\section{Existing Algorithm for LPBV Problems}

Step 1: If R.H.S of any constraint is negative, make it positive by multiplying the constraint by -1 .

Step 2: Convert the inequalities of the constraints into equations by the addition of suitable slacks and or surplus variables and obtain an initial basic feasible solution.

Step 3: If any variable is at a positive lower bound, it should be substituted at its lower bound.

Step 4: Calculate the net evaluation $\Delta_{j}=C_{j}-Z_{j}$. For a maximization problem if $\Delta_{j} \leq 0$ for the non-basic variables at their upper bound, optimum basic feasible solution is attained. It not, go to step-5. Reverse is true for a minimization problem.

Step 5: Select the most positive $\Delta_{j}=C_{j}-Z_{j}$.

Step 6: Let $x_{j}$ be a non-basic variable at zero level which is selected to enter the solution. Compute the quantities,

$$
\begin{gathered}
x_{j} \leq \theta_{1}=\min _{i}\left\{\frac{\left(B^{-1} b\right)_{i}}{\left(B^{-1} P_{j}\right)_{i}} \mid\left(B^{-1} P_{j}\right)_{i}>0\right\} \text { or } \\
\theta_{1}=\min _{i}\left\{\frac{\left(B^{-1} b\right)_{i}}{\left(B^{-1} P_{j}\right)_{i}} \mid\left(B^{-1} b\right)_{i}<0 \&\left(B^{-1} P_{j}\right)_{i}<0\right\} \\
x_{j} \leq \theta_{2}=\min _{i}\left\{\frac{\left(B^{-1} b\right)_{i}-\left(U_{B}\right)_{i}}{\left(B^{-1} P_{j}\right)_{i}} \mid\left(B^{-1} P_{j}\right)_{i}<0\right\}
\end{gathered}
$$

Final condition is satisfied simply it $x_{j} \leq u_{j}$ and $\theta=$ $\min \left(\theta_{1}, \theta_{2}, u_{j}\right)$, where $\theta=$ value of the entering variable and $u_{j}$ is the upper bound for the variable $x_{j}$. Let $\left(X_{B}\right)_{r}$ be the leaving variable corresponding to $\theta=\min \left(\theta_{1}, \theta_{2}, u_{j}\right)$ and then we have the following rules:

Rule 1: If $\theta=\theta_{1},\left(X_{B}\right)_{r}$ leaves the basic solution (because non-basic) at level zero and $x_{j}$ enter by using the regular row operation of the simplex method.

Rule 2: If $\theta=\theta_{2},\left(X_{B}\right)_{r}$ leaves the basic solution at level zero and $x_{j}$ enters then $\left(X_{B}\right)_{r}$ being non-basic at its upper bound must be substituted out by using $\left(X_{B}\right)_{r}=$ $\left(U_{B}\right)_{r}-\left(X^{\prime}{ }_{B}\right)_{r}$, where $0 \leq\left(X^{\prime}{ }_{B}\right)_{r} \leq\left(U_{B}\right)_{r}$.

Rule 3: If $\theta=u_{j}, x_{j}$ is substituted at its upper bound $u_{j}-x_{j}^{\prime}$ but remain non-basic. 
A tie among $\theta_{1}, \theta_{2}$ and $u_{j}$ may be broken arbitrarily. However, it is preferable to implement the rule for $\theta=u_{j}$ because it entails less computation.

In the next section, we will develop a method for solving quasi-concave QPBV problems and also illustrate the solution procedure with a number of numerical examples.

\section{Proposed Method for Quasi-Concave QPBV Problems}

In this section, we propose a new method namely, $\mathrm{OSM}^{22}$ to finding an optimal solution to the quasi-concave QPBV problem and also include numerical examples to demonstrate our method.

The proposed method proceeds as follows:

Step 1: Construct two single objective linear programming problems with bounded variables namely, the problem $(\mathrm{P} 1)$ and the problem $(\mathrm{P} 2)$ from the given problem $(\mathrm{P})$.

Step 2: Compute the optimal solution to the problem (P1) using the LPBV algorithm. Let the optimal solution to the problem (P1) be $X_{0}$ and the maximum value of $Z_{1}(X)=$ $Z_{1}\left(X_{0}\right)$.

Step 3: Use the optimal table of the problem (P1) as an initial bounded variables simplex table for the problem (P2), and obtain a sequence of basic feasible solutions to the problem (P2) by the LPBV algorithm.

Step 4: Let $\left\{X_{n}\right\}$ be a sequence of basic feasible solutions to the problem (P2) obtained in Step 3. If $Z\left(X_{k}\right) \leq Z\left(X_{k+1}\right)$ for all $k=0,1,2, \cdots \cdots, n$ and $X_{n+1}$ is an optimal solution to the problem (P2) for some $n$, stop the computation process and then, go to Step 5 or Step 6.

Step 5: If $Z\left(X_{k}\right) \leq Z\left(X_{k+1}\right)$ for all $k=0,1,2, \cdots \cdots, n$ and $Z\left(X_{n+1}\right) \geq Z\left(X_{n+2}\right)$, then stop the computation process and then, go to Step 6 .

Step 6: $X_{n+1}$ is an optimal solution to the problem $(\mathrm{P})$ and the maximum value of $Z(X)=Z\left(X_{n+1}\right)$ by the Theorem 3.1 .

Step 7: If the constraints set is not in a canonical form then follows the following sub-steps:

Sub-step 1: Introduce artificial variables wherever it is required. Consider all variables are non-negative.

Sub-step 2: Then write it as an artificial linear objective function as in minimization type (minimization: $w_{1}+w_{2}+$ $\cdots)$. In phase-I, solve the problem as a regular linear program.

Sub-step 3: Compute relative profit factor

$$
\Delta_{j}=c_{j}-z_{j}
$$

Sub-step 4: For minimization problem, if $\Delta_{j} \geq 0$ for all nonbasic variables and the objective function (i.e. minimization: $\left.w_{1}+w_{2}+\cdots\right)$ equal to zero and also all artificial variables leave the basis then the original quasi-concave QPBV problem has a basic feasible solution. If, not then the problem has no optimal solution.

Sub-step 5: When it is feasible then remove all columns corresponding to the artificial variables and construct a new table to solve original quasi-concave QPBV problem with initial solution found at the end of phase-I. Then, repeat step 1 to step 6.

\section{Remark 4.1}

The maximum value for $(n+1)$ is less than or equal to the number of the iterations to obtain the optimal solution to the problem (P2) by the LPBV algorithm.

\section{Numerical Example 1}

Consider the following quasi-concave QPBV problem:

Max, $Z=\left(2 x_{1}+3 x_{2}+12\right) \cdot\left(x_{1}+3 x_{2}+6\right)$

Subject to, $x_{1}+2 x_{2} \geq 10$

$$
\begin{aligned}
& 2 x_{1}+3 x_{2} \leq 60 \\
& 5 \leq x_{1} \leq 15, \quad 4 \leq x_{2} \leq 30
\end{aligned}
$$

\section{Solution: Using Our Proposed Method}

In our problem, the constraints are not in a canonical form. So apply step 7 . Then our problem becomes:

Min, $U=w$

Subject to, $\quad x_{1}+2 x_{2}-s_{1}+w=10$

$$
2 x_{1}+3 x_{2}+s_{2}=60
$$$$
x_{1}, x_{2}, s_{1}, s_{2}, w \geq 0
$$

Table 1. Final table for finding basic variables

\begin{tabular}{|c|c|c|c|c|c|c|c|}
\hline \multirow{2}{*}{$c_{B}$} & $c_{j}$ & 0 & 0 & 0 & 0 & 1 & \\
\cline { 2 - 8 } & Basis & $x_{1}$ & $x_{2}$ & $s_{1}$ & $s_{2}$ & $w$ & $b$ \\
\hline 0 & $x_{2}$ & $\cdot 5$ & 1 & $-\cdot 5$ & 0 & $\cdot 5$ & 5 \\
\hline 0 & $s_{2}$ & $\cdot 5$ & 0 & $1 \cdot 5$ & 1 & $-1 \cdot 5$ & 45 \\
\hline \multicolumn{2}{|c|}{$c_{j}-z_{j}$} & 0 & 0 & 0 & 0 & 1 & $U=0$ \\
\hline
\end{tabular}

Since all $c_{j}-z_{j} \geq 0$ and Min $U=0$ and all artificial variables leave the basis. So the original quasi-concave QPBV problem has a basic feasible solution. After the above calculation, we take

$$
\begin{gathered}
x_{1}=5+y_{1}, 0 \leq y_{1} \leq 10 \text { and } \\
x_{2}=4+y_{2}, 0 \leq y_{2} \leq 26 .
\end{gathered}
$$

Now, solve the original quasi-concave QPBV problem with initial solution found at the end of phase-I. Then the original quasi-concave QPBV problem becomes

(P) Max, $z=\left(2 y_{1}+3 y_{2}+34\right) \cdot\left(y_{1}+3 y_{2}+23\right)$

Subject to, $\begin{aligned} \frac{1}{2} y_{1}+y_{2}-\frac{1}{2} s_{1} & =-\frac{3}{2} \\ \frac{1}{2} y_{1}+\frac{3}{2} s_{2} & =\frac{85}{2}\end{aligned}$

$0 \leq y_{1} \leq 10,0 \leq y_{2} \leq 26$ and $s_{1}, s_{2} \geq 0$

Now, from the problem (P), two single objective linear programming problems are constructed as follows:

(P1) Max, $z^{1}=\left(2 y_{1}+3 y_{2}+34\right)$

Subject to, $\frac{1}{2} y_{1}+y_{2}-\frac{1}{2} s_{1}=-\frac{3}{2}$

$$
\frac{1}{2} y_{1}+\frac{3}{2} s_{2} \quad=\frac{85}{2}
$$

$0 \leq y_{1} \leq 10,0 \leq y_{2} \leq 26$ and $s_{1}, s_{2} \geq 0$ and 
(P2) $\operatorname{Max}, z^{2}=\left(y_{1}+3 y_{2}+23\right)$

$$
\begin{aligned}
& \text { Subject to, } \quad \frac{1}{2} y_{1}+y_{2}-\frac{1}{2} s_{1}=-\frac{3}{2} \\
& \frac{1}{2} y_{1}+\frac{3}{2} s_{2} \quad=\frac{85}{2} \\
& 0 \leq y_{1} \leq 10,0 \leq y_{2} \leq 26 \text { and } s_{1}, s_{2} \geq 0
\end{aligned}
$$

Table 2.1. Initial table for $P 1$

\begin{tabular}{|c|c|c|c|c|c|}
\hline $\begin{array}{c}c_{B} \\
\downarrow\end{array}$ & $c_{j} \rightarrow$ & 2 & 3 & 0 & 0 \\
\cline { 2 - 6 } & Basis $\downarrow$ & $y_{1}$ & $y_{2}$ & $s_{1}$ & $s_{2}$ \\
\hline 3 & $\begin{array}{c}y_{2} \\
=-3 / 2\end{array}$ & $1 / 2$ & 1 & $-1 / 2$ & 0 \\
\hline 0 & $s_{2}=85 / 2$ & $1 / 2$ & 0 & $3 / 2$ & 1 \\
\hline$z^{1}=25$ & $c_{j}-z_{j}^{1}$ & $1 / 2$ & 0 & $3 / 2 \uparrow$ & 0 \\
\hline
\end{tabular}

Here, $\theta_{1}=\min \left\{\frac{-3 / 2}{-1 / 2}, \frac{85 / 2}{3 / 2}\right\}=3, \theta_{2}=\infty$, since $s_{1} \geq 0$. So $\theta=\min \left\{\theta_{1}, \theta_{2}, U_{1}\right\}=3=\theta_{1}$.

Table 3.1.

\begin{tabular}{|c|c|c|c|c|c|}
\hline \multirow{2}{*}{$\begin{array}{c}c_{B} \\
\downarrow\end{array}$} & $c_{j} \rightarrow$ & 2 & 3 & 0 & 0 \\
\cline { 2 - 6 } & Basis $\downarrow$ & $y_{1}$ & $y_{2}$ & $s_{1}$ & $s_{2}$ \\
\hline 0 & $s_{1}=3$ & -1 & -2 & 1 & 0 \\
\hline 0 & $s_{2}=38$ & 2 & 3 & 0 & 1 \\
\hline$z^{1}=34$ & $c_{j}-z_{j}^{1}$ & 2 & $3 \uparrow$ & 0 & 0 \\
\hline
\end{tabular}

Here, $\theta_{1}=12 \cdot 66, \theta_{2}=\infty$, since $s_{1} \geq 0$. So $\theta=$ $\min \left\{\theta_{1}, \theta_{2}, U_{2}\right\}=12 \cdot 66=\theta_{1}$.

Table 4.1. Optimal table

\begin{tabular}{|c|c|c|c|c|c|}
\hline \multirow{2}{*}{$\begin{array}{c}c_{B} \\
\downarrow\end{array}$} & $c_{j} \rightarrow$ & 2 & 3 & 0 & 0 \\
\cline { 2 - 6 } & Basis $\downarrow$ & $y_{1}$ & $y_{2}$ & $s_{1}$ & $s_{2}$ \\
\hline 0 & $s_{1}=85 / 3$ & $1 / 3$ & 0 & 1 & $2 / 3$ \\
\hline 3 & $y_{2}=38 / 3$ & $2 / 3$ & 1 & 0 & $1 / 3$ \\
\hline$z^{1}=72$ & $c_{j}-z_{j}^{1}$ & 0 & 0 & 0 & -1 \\
\hline
\end{tabular}

Since all $\Delta_{j} \leq 0$ in Table-4.1, this table gives the optimal solution.

Table 2.2. Initial table for $\mathbf{P 2}$

\begin{tabular}{|c|c|c|c|c|c|}
\hline$d_{B}$ & $d_{j} \rightarrow$ & 1 & 3 & 0 & 0 \\
\cline { 2 - 6 }$\downarrow$ & Basis $\downarrow$ & $y_{1}$ & $y_{2}$ & $s_{1}$ & $s_{2}$ \\
\hline 3 & $\begin{array}{c}y_{2} \\
=-3 / 2\end{array}$ & $1 / 2$ & 1 & $-1 / 2$ & 0 \\
\hline 0 & $s_{2}=85 / 2$ & $1 / 2$ & 0 & $3 / 2$ & 1 \\
\hline$z^{2}=14$ & $d_{j}-z_{j}^{2}$ & $-1 / 2$ & 0 & $3 / 2 \uparrow$ & 0 \\
\hline
\end{tabular}

Here, $\theta_{1}=\min \left\{\frac{-3 / 2}{-1 / 2}, \frac{85 / 2}{3 / 2}\right\}=3, \theta_{2}=\infty$, since $s_{1} \geq 0$. So $\theta=\min \left\{\theta_{1}, \theta_{2}, \mathrm{U}_{1}\right\}=3=\theta_{1}$.

Table 3.2.

\begin{tabular}{|c|c|c|c|c|c|}
\hline$d_{B}$ & $d_{j} \rightarrow$ & 1 & 3 & 0 & 0 \\
\cline { 2 - 6 }$\downarrow$ & Basis $\downarrow$ & $y_{1}$ & $y_{2}$ & $s_{1}$ & $s_{2}$ \\
\hline 0 & $s_{1}=3$ & -1 & -2 & 1 & 0 \\
\hline
\end{tabular}

\begin{tabular}{|c|c|c|c|c|c|}
\hline 0 & $S_{2}=38$ & 2 & 3 & 0 & 1 \\
\hline$z^{2}=23$ & $d_{j}-z_{j}^{2}$ & 1 & $3 \uparrow$ & 0 & 0 \\
\hline
\end{tabular}

Here, $\theta_{1}=12 \cdot 66, \theta_{2}=\infty$, since $s_{1} \geq 0$. So $\theta=$ $\min \left\{\theta_{1}, \theta_{2}, U_{2}\right\}=12 \cdot 66=\theta_{1}$.

Table 4.2 Optimal table

\begin{tabular}{|c|c|c|c|c|c|}
\hline$d_{B}$ & $d_{j} \rightarrow$ & 1 & 3 & 0 & 0 \\
\cline { 2 - 6 }$\downarrow$ & Basis $\downarrow$ & $y_{1}$ & $y_{2}$ & $s_{1}$ & $s_{2}$ \\
\hline 0 & $s_{1}=85 / 3$ & $1 / 3$ & 0 & 1 & $2 / 3$ \\
\hline 3 & $\begin{array}{c}y_{2} \\
=38 / 3\end{array}$ & $2 / 3$ & 1 & 0 & $1 / 3$ \\
\hline$z^{2}=61$ & $d_{j}-z_{j}^{2}$ & -1 & 0 & 0 & -1 \\
\hline
\end{tabular}

Since all $\Delta_{j} \leq 0$ in Table-4.2, this table gives the optimal solution.

The optimal solution in term of the original variables $x_{1}, x_{2}$ is found as follows: $x_{1}=5+y_{1}=5+0=5$ and $x_{2}=4+$ $y_{2}=4+(38 / 3)=50 / 3$ with $Z_{\max }=z^{1} \cdot z^{2}=4392$.

\section{Numerical Example 2}

Consider the following quasi-concave QPBV problem:

(P) Max, $Z=\left(0.07 x_{1}+0.09 x_{2}\right) \cdot\left(x_{1}+x_{2}+100\right)$

Subject to, $\quad x_{1}+x_{2} \leq 100000$

$$
\begin{aligned}
& x_{1}-2 x_{2} \geq 0 \\
& x_{1} \geq 0,0 \leq x_{2} \leq 30000
\end{aligned}
$$

\section{Solution: Using Our Proposed Method}

The following two LP problem can be obtained from the given problem $(\mathrm{P})$ :

$$
\begin{array}{cc}
\text { (P1) Max, } Z_{1}= & \left(0.07 x_{1}+0.09 x_{2}\right) \\
\text { Subject to, } & x_{1}+x_{2} \leq 100000 \\
& x_{1}-2 x_{2} \geq 0 \\
& x_{1} \geq 0,0 \leq x_{2} \leq 30000 \\
& \text { and } \\
\text { (P2) Max, } Z_{2}= & \left(x_{1}+x_{2}+100\right) \\
\text { Subject to, } & x_{1}+x_{2} \leq 100000 \\
& x_{1}-2 x_{2} \geq 0 \\
& x_{1} \geq 0,0 \leq x_{2} \leq 30000
\end{array}
$$

Now by LPBV method, the optimal solution to the problem $(\mathrm{P} 1)$ is $x_{1}=70000, x_{2}=30000$ with $\operatorname{Max} Z_{1}=7600$.

Now, by the step 3 of the proposed method, the solution to the problem (P2) by LPBV method is given below:

\begin{tabular}{|c|c|c|c|c|}
\hline It. & Solution & Max & Max & Max \\
No & $\left(x_{1}, x_{2}, s_{1}, s_{2}, s_{3}\right)$ & $Z_{1}$ & $Z_{2}$ & $\begin{array}{c}Z= \\
Z_{1} Z_{2}\end{array}$ \\
\hline 0 & $(60000,30000,10000,0,0)$ & 6900 & 90100 & 621690000 \\
\hline 1 & $(70000,30000,0,10000,0)$ & 7600 & 100100 & 760760000 \\
\hline 2 & $(0,0,100000,0,30000)$ & 0 & 100 & 0 \\
\hline 3 & $(100000,0,0,100000,30000)$ & 7000 & 107000 & 700700000 \\
\hline 4 & $(0,0,100000,0,30000)$ & 0 & 100 & 0 \\
\hline 5 & $(0,0,100000,0,30000)$ & 0 & 100 & 0 \\
\hline
\end{tabular}

Since the $1^{\text {st }}$ iteration table is optimal and by the step 4 of the proposed method, the optimal solution to the given QPBV problem is $x_{1}=70000, x_{2}=30000$ with Max $Z=760760000$.

\section{Algorithm and Computer Technique}


In this section, we present algorithm and computational technique for solving LPBV problems. In this study, we extend that method for solving quasi-concave QPBV problems.

\section{Algorithm for solving LPBV problems}

Step 1: Express the LPBV problem to its standard form.

Step 2: Find an $m \times m$ sub-matrix of the coefficient matrix $A$ by setting $n-m$ variables equal to zero.

Step 3: Test whether the linear system of equations has unique solution or not.

Step 4: If the linear system of equations has got any unique solution, find it.
Step 5: Dropping the solutions with negative elements. Determine all basic feasible solutions.

Step 6: Calculate the values of the objective function for the basic feasible solutions found in step-5.

Step 7: For maximization of LPBV problem, the maximum value of $Z$ is the optimal value of the objection function and the basic feasible solution which yields the optimal value is the optimal solution.

\section{Computer code for solving LPBV problems}

In this section, we present a computer technique for solving LPBV problems using the programming language MATHEMATICA ${ }^{20,21}$.

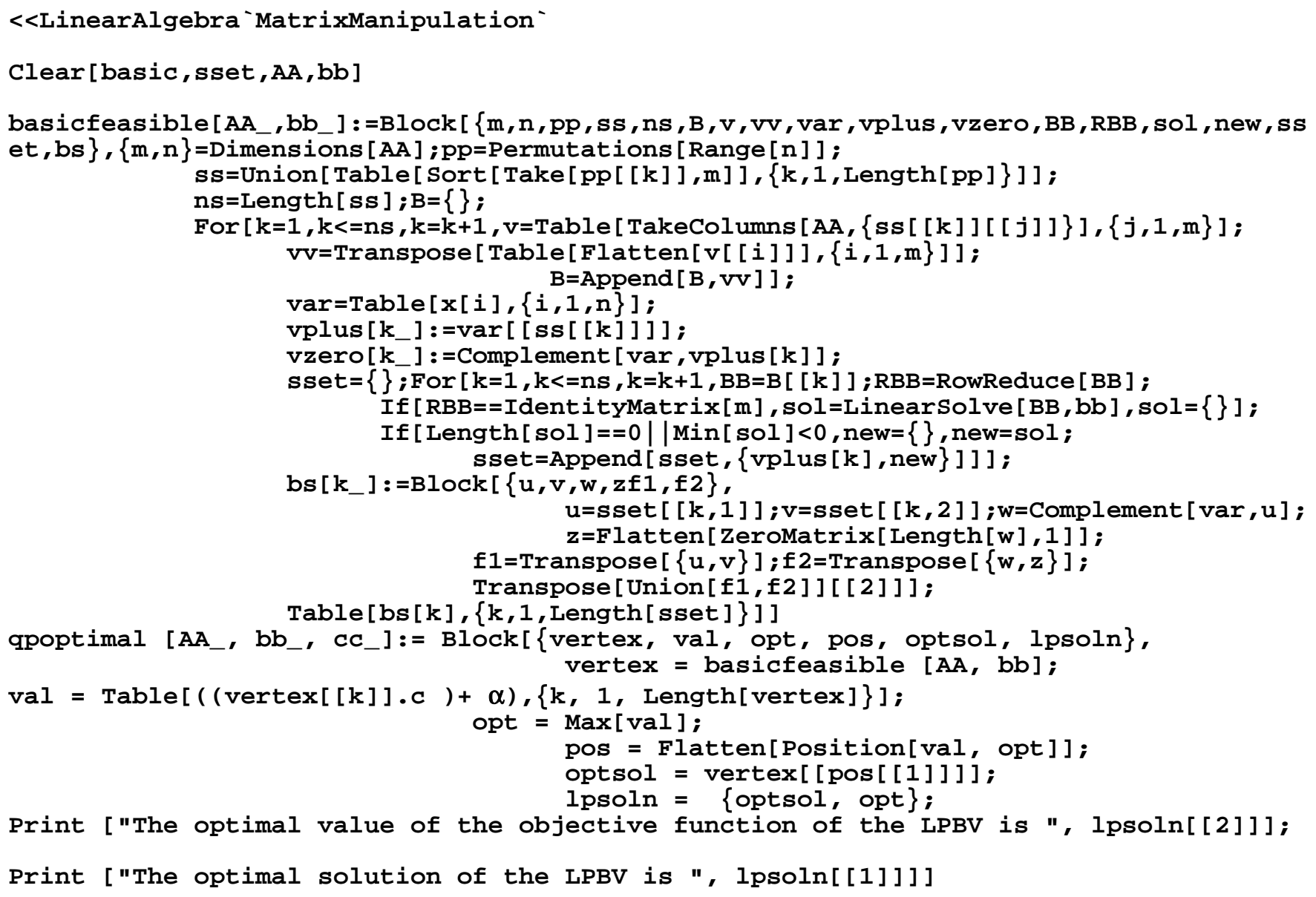

\section{Numerical Examples}

In this section, solve the same problems which were solved in section IV by above computer technique.

\section{Input for Numerical Example 1}

For problem (P1)

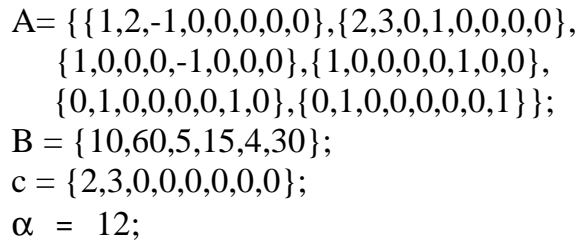

basicfeasible[A,b] qpoptimal[A, b, c] For problem $(\mathrm{P} 2)$

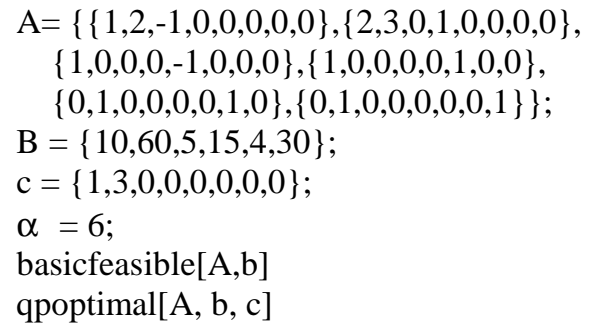

Output for Numerical Example 1 
For problem (P1)

The possible all basic solution is:

$\{\{15,4,13,18,10,0,0,26\},\{5,4,3,38,0,10,0,26\},\{15,10,25,0,1$ $0,0,6,20\},\{5,50 / 3,85 / 3,0,0,10,38 / 3,40 / 3\}\}$

The optimal value of the objective function of the LPBV is 72

The optimal solution of the LPBV is $\{15,10,25,0,10,0,6,20\}$

For problem (P2)

The possible all basic solution is:

$\{\{15,4,13,18,10,0,0,26\},\{5,4,3,38,0,10,0,26\},\{15,10,25,0,1$ $0,0,6,20\},\{5,50 / 3,85 / 3,0,0,10,38 / 3,40 / 3\}\}$

The optimal value of the objective function of the LPBV is 61

The optimal solution of the LPBV is

$\{5,50 / 3,85 / 3,0,0,10,38 / 3,40 / 3\}$

So, the optimal solution to the given QPBV problem is $x_{1}=5, x_{2}=\frac{50}{3}$ with $\operatorname{Max} Z=4392$.

Input for Numerical Example 2

For problem (P1)

Clear[A,b,c]

$A=\{\{1,1,1,0,0\},\{1,-2,0,-1,0\},\{0,1,0,0,1\}\}$;

$\mathrm{b}=\{100000,0,30000\} ; \mathrm{c}=\{.07, .09,0,0,0\}$;

$\alpha=0$;

basicfeasible[A,b]

qpoptimal[A, b, c]

For problem $(\mathrm{P} 2)$

$\mathrm{A}=\{\{1,1,1,0,0\},\{1,-2,0,-1,0\},\{0,1,0,0,1\}\} ;$

$\mathrm{b}=\{100000,0,30000\} ; \mathrm{c}=\{1,1,0,0,0\}$;

$\alpha=100$

basicfeasible[A,b]

qpoptimal[A, b, c]

Output for Numerical Example 2

For problem (P1)

The possible all basic solution is:

$\{\{60000,30000,10000,0,0\},\{70000,30000,0,10000,0\},\{0,0$, $100000,0,30000\},\{100000,0,0,100000,30000\},\{0,0,100000$, $0,30000\},\{0,0,100000,0,30000\}\}$

The optimal value of the objective function of the LPBV is 7600

The optimal solution of the LPBV is

$\{70000,30000,0,10000,0\}$

For problem (P2)

The possible all basic solution is:

$\{\{60000,30000,10000,0,0\},\{70000,30000,0,10000,0\},\{0,0$, $100000,0,30000\},\{100000,0,0,100000,30000\},\{0,0,100000$, $0,30000\},\{0,0,100000,0,30000\}\}$
The optimal value of the objective function of the LPBV is 100100

The optimal solution of the LPBV is $\{70000,30000,0,10000,0\}$

So, the optimal solution to the given QPBV problem is $x_{1}=70000, x_{2}=30000$ with $\operatorname{Max} Z=760760000$.

We observed that the result obtained by computer technique is completely identical with the result obtained by our proposed method for solving quasi-concave QPBV problems. In our computer technique, we just had to compute the coefficient matrix $A$, right hand side constant $b$, cost coefficient vectors $c$ and $d$ and the constants $\alpha$ and $\beta$ and easily obtained the optimal solution. Also we observed that our computer oriented method can solve any LPBV problems.

\section{Conclusions}

The aim of this paper was to develop an easy technique for solving quasi-concave QPBV problems. So in this paper, we developed a new method namely, OSM based on LPBV algorithm for finding an optimal solution to a quasi-concave QP problem with bounded variables in which the objective function involves the product of two indefinite factorized linear functions and the constraint functions are in the form of linear inequalities. We illustrate some numerical examples to demonstrate our proposed method. We also developed a computer technique by using programming language MATHEMATICA for LPBV problems. We therefore, hope that our proposed method and computer technique can be used as an effective tool for solving quasiconcave QPBV problems and hence our time and labor can be saved.

\section{References}

1. Wolfe, P., 1959. The simplex method for quadratic programming, Econometrica, 27, 3. 382-398.

2. Beale, E.M.L., 1959. On quadratic programming, Naval Research Logistics Quarterly, 6, 227-243.

3. Frank, M., P. Wolfe, 1956. An algorithm for quadratic programming, Naval Research Logistics Quarterly, 3, 95-110.

4. Shetty, C.M., 1963. A simplified procedure for quadratic programming, Operations Research, 11, 248-260.

5. Lemke, C.E., 1965. Bi-matrix equilibrium points and mathematical programming, Management Science, 11, 681689.

6. Best, M.J., K. Ritter K, 1988. A quadratic programming algorithm, Zeitschrift for Operational Research,32, 271-297.

7. Theil, H., C. Van de Panne, 1961. Quadratic programming as an extension of conventional quadratic maximization, Management Science, 7, 1-20.

8. J.C.G., Boot, 1961. Notes on quadratic programming: The kuhn-Tucker and Theil-van de Panne conditions, degeneracy and equality constraints, Management Science, 8, 85-98.

9. Fletcher, R., 1971. A general quadratic programming algorithm, J. Inst., Maths. Applics, 7, 76-91.

10. Swarup, K., 1966. Quadratic programming, CCERO (Belgium), 8, 132-136. 
11. Gupta, A.K., J.K. Sharma, 1983. A generalized simplex technique for solving quadratic programming problem, Indian Journal of Technology, 21, 198-201.

12. Moraru, V., 1997. An algorithm for solving quadratic programming problems, Computer science Journal of Moldova, 5, 223-235.

13. Moraru, V., 2000. Primal-dual method for solving convex quadratic programming problems, Computer science Journal of Moldova, 8, 209-220.

14. Jensen, D.L., A.J. King, 1992. A decomposition method for quadratic programming, IBM Systems Journal, 31, 39-48.

15. Bazaraa, M., H. Sherali, C.M. Shetty, 2006. Nonlinear programming: Theory and algorithm (John Wiley, New York).

16. Whinston, A., 1956. The Bounded Variable Problem- An Application of The Dual Method For Quadratic Programming, Naval Research Logistics Quarterly, 12(2), 173-179.

17. Hamadhy, A. Taha, 2007. Operation Research: An Introduction, $8^{\text {th }}$ edition, Pearson Prentice Hall, Pearson Education, Inc., Upper Saddle River, NJ 07458.

18. Hasan, M. B., 2012. A Technique for solving Special Type Quadratic Programming Problems, The Dhaka University journal of science, $\mathbf{6 0}(\mathbf{2}), 209-215$.
19. Gupta, P.K., D.S. Hira, Problem in Operation Research, S. Chand \& Company Ltd, Ram Nagar, New Delhi-110055.

20. Don, Eugene, 2001. Theory and Problems of Mathematica, Schaum's Outline Series, McGraw-Hill, New York San Francisco Washington, D.C.

21. Wolfram, S., 2000. Mathematica, Addision-wesley Publication Company, Melno Park, California, New York.

22. Jayalakshmi, M., Pandian P., 2014, A method for solving quadratic programming problems having linearly factorized objective function, International Journal Of Modern Engineering Research, 4, 20-24.

23. Ezio Marchi, 2008, When is the product of two concave functions concave?, IMA Preprint Series \# 2204, Institute for Mathematics and its Applications, University of Minnesota, 1-8. 